\title{
Preliminary Research and Overview of Blockchain Application in Generation and Transmission System
}

\author{
Cuihui Yan*, Fang Liü, Zhiqiang $\mathrm{Luo}^{2}$, Junfeng Yang${ }^{2}$, Qiang Ding ${ }^{1}$ and $\mathrm{Min}^{\mathrm{L}} \mathrm{i}^{3}$ \\ ${ }^{1}$ Power System Automation Department, China Electric Power Research Institute, Haidian District, Beijing 100192, China \\ ${ }^{2}$ National Power Dispatching and Control Center, State Grid Corporation of China, Beijing, China \\ ${ }^{3}$ Sichuan Electric Power Dispatching and Control Center, State Grid SICHUAN Electric Power Company, State Grid, Corporation \\ of China, Chengdu, China \\ ${ }^{*}$ Corresponding author
}

\begin{abstract}
Although China's electricity market reform has achieved some results, it still not fully enter into the dispatch scheduling system. The new born technology blockchain has been widely realized, it also appeared many research in energy internet field and received some achievement. In this case, this paper attempted to introduce the blockchain to the generating and transmission scheduling system of dispatch scheduling, it make some theoretical analysis on blockchain application in dispatch scheduling fields. Firstly, it introduced the definition and characteristic of blockchain, and analyzed the technical framework of blockchain and some application scenarios. Secondly, it discussed the application feasibility of blockchain in generating and transmission scheduling system, and summarized the effect and problems may come out with blockchain. In the end, it expected and prospected the application of blockchain in generating and transmission scheduling system in dispatch scheduling fields. It also hope to attract some researchers attention to research the blockchain in dispatch scheduling field to give full play of the decentralization, transparency and traceability superiority of blockchain.
\end{abstract}

Keywords-generating and transmission scheduling; power generation capacity; blockchain; information management; traceability

\section{INTRODUCTION}

In recent years, it controlled by the state grid electric power dispatching center, China Electric Power Research Institute, State Grid Electric Power Research Institute together to participate in the development of the smart grid dispatching control system, which has put into use in provincial scheduling center and above all dispatching centers. The system used an oriented architecture eservice which follow the idea of "horizontal integration, cut-through in lengthways and maintain in source, sharing in the whole network" as in refers [1].

As a new technology, the development of blockchain has received wide attention from the whole society, and it has already had a preliminary application in the finance field. The application of blockchain in the field of energy has already begun a preliminary exploration as in refers [2]. The infers [3] based on the concept of energy system in the central blockchain, which with a brief overview of the research status in the field of energy blockchain, and business model from P2P trading, electric vehicle charging, physical information security and energy Internet facing the blockchain future with a preliminary outlook. In refers [4],it analyzed the application scenarios and business models of blockchain in the field of energy generation, transmission, distribution, utilization and storage, and puts forward some problems and suggestions for developing energy blockchain in China. The refers [5] based on the basic principle of blockchain technology and typical characteristics, analyzed and summarized the application of block chain technology in the energy Internet from 3 dimensions of function, subject, attribute, energy, and information from the perspective of value chain, describes the block technology for energy sources, the Internet Network Holland, storage of different themes, in the measurement certification, market transaction, coordination organization, energy finance in different sectors play a role of and prospects for the typical application of blockchain technology in energy transformation in china. In The refers[6] it proposes the application of a blockchain technology in ancillary service, and it has carried on the analysis from the consensus contract, business accounting, intelligent contract and other aspects of the blockchain technology application , it summarized the limitations of the current blockchain technology application in power market ancillary service areas. In refers [7], it proposed a mechanism framework for distributed decision making and autonomous operation of energy Internet based on blockchain technology. The key technologies of energy Internet development under blockchain framework were put forward from block chain and energy Internet point of view. The study on blockchain in energy internet has been proceed positively, and has become the focus of energy internet research, it has been started in the power market and less study in the intra-day transmission schedule areas.

\section{BLOCKCHAIN}

Blockchain is a chain database formed by an orderly connection of data blocks. The connected data blocks are protected by cryptography way, the database is maintained by all nodes in the network which is save together, preserved together and has the characteristics of no forgery and tampering, it can solves the trust problem among the subjects in the complex system. Blockchain used the decentralized and detrust method to maintain a reliable database technical scheme as in refers [3]. This coincides with the idea of the reliability of the scheduling database and the idea of centralization. 


\section{A. Conception of Blockchain}

The blockchain consisted of series of data blocks which is linked with cryptographic methods according to the time sequence, it make up of a message chain (that is database). In the application of bitcoin as in refer [16], each block recoded the transaction information crossing a certain time range and published by the participating nodes. The transaction information includes the identification of the sending and receiving parties, the transaction amount, the transaction time, and other additional information. Each data block contains either the above transaction data, or other information such as block ID, timestamp, and connection with the previous block, so that it can be linked to a chain [5] which records all the historical transaction information of the entire network. These data blocks are broadcasted to the entire shared network in real time, once the message recognized and added to the chain, they are non-denied and difficult to tamper with, so the entire database can be ensured its transparency and reliability.

It refers [6] described the conception of blockchain from the narrow sense and the generalized two aspects, in narrow sense, it is defined as a chain data structure which is connected by data blocks in chronological order, and the cryptography guarantees its non-tampering and non-fabricated of the distributed account, in generalized sense, this technology is broadened to a new application mode of computer technology in the Internet era, such as distributed data storage, point to point transmission, consensus mechanism, encryption algorithm and so on.

\section{B. Feature of the Blockchain}

The blockchain has four technical features as referred in $[7,8]$, which is decentralized, transparency, automation of contract execution and traceability. The decentralized means the whole blockchain network without a compelled control center, each node in the network have the same rights and obligations, the data in many nodes is backup to each other, therefore, the data damage or exception of any node does not affect the operation of the whole data system, that is, there is no single center unit can manipulate the data unilaterally, which makes the data storage based on blockchain has high reliability and robustness.

The transparency refers to the whole blockchain system operation rules are open and transparent, the blockchain record information made redundancy back-up in multiple nodes, the information update need common verified in multiple nodes, thus the data exchange between nodes is off trust, one node cannot deceive the other nodes.

The contract executed automatically refers to an intelligent contract which can be set a series embedded writing software code using blockchain, it is regulated the obligations to be performed by each party and the criteria for the execution in an intelligent contract, the blockchain system will judge the contract execution conditions automatically, When all the conditions are satisfied, the blockchain system will enforce the contract terms automatically. This on one hand improves the contract implementation efficiency, and more importantly, the implementation of the contract can be executed effectively without a strong third party supervision.
Traceability means that the records added to the blockchain are stored permanently, and each transaction record in the blockchain is bound to the trader information, the complete transmission subject can be recorded and traced completely, and it cannot be destroyed or tampered. Which has brought convenience to the regulation of the transaction.

\section{APPLICATION FEASIBILITY OF BLOCKCHAIN IN SCHEDULE SYSTEM}

From the characteristics of blockchain technology and schedule plan, there are many similarities between the two. This also lays a good foundation for the cooperation of the two. At the same time, the application of blockchain technique in scheduling is thus in the attempt stage, such research is still very rare, but the characteristics of blockchain has superior application basement based on scheduling plan, provide a good solution for the scheduling of the problems encountered in the development process.

\section{A. The Function of Blockchain in Intra-Generation Power and Power Generation Capacity Declaration System}

Energy flow and information flow are integrated with each other, and transmission is one of the basic features of the transmission and transmission planning system. The intraday transmission schedule realized the information exchange dispatching control center within the provincial area and the power plant, in order to support the other scheduling application. It includes generation and transmission declaration with different period, transmission and transmission schedule management, generation and transmission schedule adjustment, transmission and transmission plan approval / review, generation and transmission review statistical analysis and generation and transmission release. The subordinate regional dispatch and power plant need to provide the necessary business information to the superior dispatch control center according to need, the superior dispatching control center also should offer part of the work results to subordinates regional dispatch, each dispatch can obtain needed information and make decision for business problems timely through information transfer. It can receive the intraday generation and transmission schedule information from dispatch object, verified and disposed synergistically according to the default rule. The blockchain as a shared distributed database technology, each node of blockchain can recorded data updates to the network, each involved node can obtained a whole database copy, the information in the block will be copied to all the blocks in the network, and the full network data can be synchronized as referred in [8].

The goal of information transmission in electric power is to provide secure and reliable information to the demand side using instrument according to the unified, easy and traceability method, which can support the mutual ringed business process using consistent and synchronous information data.

The nature of blockchain decentralization helps to solve some of the security problems in the physical information system. As in refers [5], only the authorized node can get the public key of other nodes and sensors in the network, so it will not be able to decrypt the data transmitted, if an attacker doesn't 
have a public key. The system node linked into the network structure in the data transmission layer as in refers [12], then the data path exist redundancy. Even if an attacker blocked data path in the network topology, the information can still be transmitted through other data path. In the application control layer, all users personal information in blockchain with absolute privacy in the system, so the leakage issues of privacy information is not exist.

The system characteristics is as followings:

1) Anti-attacking: If an attacker tries to tamper with the data in blockchain database, the attacker can't attack the centralized database because of the de centralization of the blockchain system. Since the full backup of all nodes in the whole network has a database, there are a lot of data redundancy, so the attacker must control the data excel to at least $51 \%$ data nodes in the control system to realize data tampering, which makes data tampering cost increase, data tampering possibility is greatly reduced;

2) Data confidentiality: The blockchain uses asymmetric key encryption technology to solve the harsh conditions, which greatly increases the difficulty of the attacker to seize the personal privacy of the user. Even if the nodes in the system have all the data, they can only access the data within their rights, and cannot access confidential data.

3) Self-repair toughness: Every node in the system is written a whole back of the blockchain data. Even if some nodes and paths in the system are attacked, it can ensure that the specific nodes in the system reconstruct the information needed by other paths.

\section{B. The Basic Framework of Generation and Transmission Schedule Supported by Blockchain}

The generation and transmission schedule system based on blockchain is designed to interconnect large numbers of distributed energy storage devices, distributed information collection and transmission systems, and all kinds of power set nodes, it aims at establishing a highly integrated generation and transmission scheduling system of dispatching schedule with the characteristic of information complemented in crossrange and information coordinated in vertical, which is linked in power information transmission and used internet thinking and technological transformation. Blockchain is a data structure formed by an orderly inked blocks as in refers [5], the block is a basic unit of blockchain, and is also the collect of relevant information and discipline. In the generation and transmission schedule, the generation unit declaration information, generating statistics, generation capacity, coal consumption curve, power generation equipment information management and other modules with each time period of generation and transmission schedule was aggregated into information blocks, which is including the power generation equipment information, the power generation capacity, the power generation capacity, the dispatching data and so on, in order to ensure the traceability of blockchain and the data non-tampering, it stamped time stamp, and the information block is linked with the irreversible time dimension and information block of the previous period, it can recorded the transmission schedule completely during each system operation period. The basic framework of the transmission and transmission schedule supported by the blockchain is shown in Figure I.

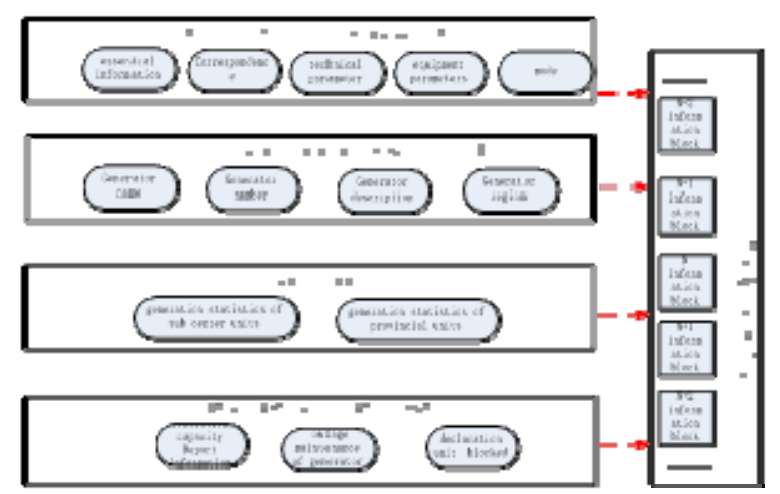

FIGURE I. THE BASIC FRAMEWORK OF THE TRANSMISSION AND TRANSMISSION PLAN SUPPORTED BY BLOCKCHAIN

\section{THE APPLICATION PROSPECT OF THE GENERATION AND TRANSMISSION SCHEDULE SYSTEM}

The generation and transmission schedule includes multiple modules, such as generating unit information management, generating capacity management, generating power statistics, generating capacity report and so on, the energy flow and information flow interaction are coexisted with each other, the transmission information between the upper and lower levels needed use a unified, easy to use, traceable way to transmit data safely and reliably between systems. The working process is supported by the consistent and synchronous information data and buckled with each other. It can received, verified and processed the registration information and running information of the dispatcher, and handled the information of the generation and transmission schedule information synergistically. It can put forward higher requirement for the information reliability, and the traceability of information is also essential for system maintenance.

\section{A. Develop Imagination of Blockchain}

It can shared the energy data, equipment parameters and operation data based on the information in blockchain of the generation and transmission schedule system with each module, it proposed the next time operation strategy according to information management, unit generating capacity and statistical module or multiple modules of the generation and transmission schedule as in refers [6], the running strategy proposed by each body will be sent to all the subjects of the system to participate in the decision, each decision maker takes the optimization result of the optimization objective function of the module running as the standard, it selected the optimal operation strategy and ordered the selected operation strategy centralized, then it can obtained the final optimal operation strategy. The information block with time stamp will keep the system running information well and ensure the system safe, stable and efficient operation.

A lot of work of energy internet construction has carried out with blockchain in China as in refers [2], it has established a financial trading system which characteristic is transparent, broad participation and overall confidence, and build between 
the extensive energy physical network and extensive energy information application network. It has not yet fully established the corresponding financial system in the generation and transmission schedule due to the marketization has not fully penetrated into the dispatching field, it only conceived the related information and physical layer. The financial layer is also inevitable with the development of electricity market. The so-called physical layer is the basic layer of the information of the power grid equipment. Information layer mainly includes information interaction, coordination control, distribution Unicom, co-sharing, and so on. The financial layer includes transaction trust, transparent and efficient, real-time sharing, intelligent contract and so on.

\section{B. Problems between Blockchain and Generation and Transmission Schedule System}

The existing problems between current blockchain and generation and transmission integration is conclude from three aspects, that is the generation and transmission system itself, the blockchain technology itself and the integration of block chain and generation and transmission system, which is summarized as follows:

1) At present, the application of power system planning has been more mature, the application of blockchain in the dispatching scheduling system existed big external barrier and industrial restrictions, information platform monitoring is uncertain, the corresponding risk is difficult to predict, and also no ready-made model can be used for reference.

2) there is also some technical bottlenecks in the wide application of blockchain, still some technical problems need to be solved in terms of computing efficiency, decentralization, privacy and security as in refers [19]. At present, the computing power and response speed of blockchain cannot meet the requirements of real-time computing in the application area, besides, the relevant information of all participants related to blockchain requires to synchronize storage, which requires large capacity storage system to support.

3) The application of blockchain technology in the field of scheduling planning is still in the conception stage, the dispatching system is much more elaborate, its optimal dispatch referred to the synchronization and real-time mutual communication between mutual systems of the platforms. There is still a long way to go existed in the application based on blockchain in dispatching schedule system, and also need practical verification and demonstration project operation experience.

\section{Prospect of Blockchain in the Generation and \\ Transmission System of Dispatching Schedule}

The application prospect in dispatching schedule based on blockchain is summarized, which is based on the development status of blockchain technology in the energy field, as well as the improvement of blockchain and the tendency of the dispatching schedule and the construction of future electricity market.

1) Equipment information management module: the combination of distributed and centralized mode is the typical pattern of dispatching scheduling area equipment information, the blockchain decentralization and the distributed management between each dispatching center has strong compatibility, it is contributed to realize the real-time information sharing between the centralized and distributed mode which is based on the real-time updating of blockchain, it can avoid the information management confusion in multiple applications, and reduce the duplication of the system. It can realized the transparency operation of unit only increase, deletion and equipment parameter modification, and the mutual management and mutual check and verification between the units.

2) Power generation capacity management module: there are many equipment involved in generating power, the information of the unit generating capacity curve and the generation capacity of the generator set is displayed more transparent and synchronized according to the integration of blockchain technology, it is facilitate to the customers coordination and optimization, and support to seek the generation units accurately because of the information in each blockchain is unique, the system efficiency can be improved.

3) Power generation capability report information: the formed information block can be preserved which including the operating units condition, generating capacity and the user's information, that is the formed distributed ledger and the block information chain, it has improved the transparency of transmission information. The outage maintenance declaration procedure of unit facility become more open and transparent, and the facility outage maintenance condition can be more determined.

The relevant unit generation capacity and the coal consumption data in monthly consumption information ca be formed to information chain and stored, it can realized the decentralization management and sharing of information, increased the trust degree of the users and various ranks, and also increased the security and traceability of information. The relevant unit generation capacity and the coal consumption data in monthly consumption information ca be formed to information chain and stored, it can realized the decentralization management and sharing of information, increased the trust degree of the users and various ranks, and also increased the security and traceability of information.

\section{CONCLUSION AND PROSPECT}

We has introduced blockchain into the generation and transmission system of dispatch schedule, it has proposed the framework for generation and transmission under blockchain technology, it has analyzed the feasibility of the application of blockchain technology in the generation and transmission schedule from the of blockchain technology characteristics. Blockchain technology is a new database technology, its characteristics such as decentralization, openness and transparency are consistent with the characteristics of the generation and transmission schedule, which has wide application potential under the requirements of technological change and high security of information in the future. It has provides a feasible technical choice for the establishment of a new generation of power system with the imagination of 
putting blockchain into the generation and transmission schedule. The system dispatching efficiency in the generation and transmission schedule can be solved taking advantage of the blockchain. However, there is still much more development potential in the blockchain technology accessed into the power market on dispatch schedule system construction and improvement, and also need further in-depth study, and hopes that this paper can get up the attention of relevant researchers to further deepen the research of blockchain in the power system.

\section{ACKNOWLEDGMENT}

The Paper is funded by the program of "State Grid Corp science and technology project”.

\section{REFERENCES}

[1] ZHAI Ming-yu, WANG Jin, WU Qing xi. Architecture and Key Technologies of Wide-area Distributed Real-time Database System for Power Dispatching Automation System[J]. Automation of electrical power system, 2013,37(2):67-71.

[2] YANG Dechang, ZHAO Xiaoyu, XU Zixiao, LI Yong, LI Qiang. Developing Status and Prospect Analysis of Blockchain in Energy Internet[J], Proceeding of the CSEE, 2017.37(13):3664-3671.

[3] YAN Yong, ZHAO Jun hua, WEN Fuquan, Blockchain in Energy Systems:Concept, Application and Prospect[J]. Electric Power Construction, 2017,38(2):12-20.

[4] WANG Anping,FAN Jin gang,GUO Yan lai. Application of block chain in the Energy Internet [J]. Electric power information and communication technology,2016,14(9):1-6.

[5] ZHANG Ning, WANG Yi, KANG Chongqing, CHENG Jiangnan, HE Dawei. Blockchain Technique in the Energy Internet: Preliminary Research Framework and Typical Applications[J]. Proceeding of the CSEE. 2016, 36(15):4011-4022.

[6] ZENG Ming, CHENG Jun, WANG Yuqing, LI Yuanfei, YANG Yongqi, DOU Jinyue. Primarily Research for Multi Module Cooperative Autonomous Mode of Energy Internet Under Blockchain Framework[J]. Proceeding of the CSEE. 2017.Vol.37(13):3672-3681.

[7] TAI Xue, SUN Hongbin, GUO QINGlai, Electricity transactions and congestion management based on blockchain in energy internet[J]. Power System Technology, 2016,40(12):3630-3638.

[8] GUAN Lili. Credit application based on Blockchain technology[J]. Information and computer, 2016(6):168-169.

[9] DONG Zhao hua, ZHAO Jun hua, WEN Fu quan. From Smart Grid to Energy Internet: Basic Concept and Research Framework[J]. Automation of electrical power system, 2014,38(15):1-11.

[10] CHEN Qi xing, LIU Dun nan, LIN Jin. Business Models and Market Mechanisms of Energy Internet (1)[J]. Power System Technology, 2015,39(11):3050-3056.

[11] OUYANG Xu, ZHU Xiangqian, YE Lun, YAO Jiangang. Preliminary Applications of Blockchain Technique in Large Consumers Direct Power Trading[J], Proceeding of the CSEE,2017,(37):3737-3745.

[12] LV Shi ning, YAN Yong, DING qi, WEN Fuquan, ZHAO Jun hua. Application of blockchain in energy internet: advantages, scenes and cases,[J]zhe jiang power system,2017,36(3).

[13] SHE Wei, HU Yue, YANG Xiaoyu, GAO Shan, LIU Wei, Virtual Power Plant Operation and Scheduling Model Based on Energy Blockchain Network[J], Proceeding of the CSEE,201737(13):3729-3736

[14] The development white paper of chain block chain technology and application, 2016[EB/OL.(2016-10-21)[2017-0118].http://mt.sohu.com/20161021/n47943606.shtml.

[15] LIU Dun nan, ZENG Ming, HUANG Renle. Business Models and Market Mechanisms of Energy Internet (2)[J]. power system technology, 2015. 39(11):3057-3063.
[16] CAO Yan. Energy blockchain and energy internet[J]. winder power, 2016(5):14-15

[17] WANG An ping, FAN Jingang, GUO Yanlai. The application of blockchain in energy internet[J]. electrical information and transmission technology, 2016,14(9):1-6.

[18] LI Bin, CAO Wang zhang, QI Bin. Application summarization of blockchain in power auxiliary services area[J]. Power system technology, 2017, 41(3):736-744.

[19] SHEN Xing PEI Qing qi, LIU Xue feng. Summarization of blockchain[J]. internet and information security journal.2016, 2(11):11- 